\title{
Alcohol Exposure Alters NMDAR Function in the Bed Nucleus of the Stria Terminalis
}

\author{
Thomas L Kash',4, Anthony J Baucum II', Kelly L Conrad', Roger J Colbran ${ }^{1,2,3}$ and Danny G Winder, ${ }^{*, 2,3}$ \\ 'Department of Molecular Physiology and Biophysics, Vanderbilt University School of Medicine, Nashville, TN, USA; ' ${ }^{2}$ Center for Molecular \\ Neuroscience, Vanderbilt University School of Medicine, Nashville, TN, USA; ${ }^{3}$ Kennedy Center for Research on Human Development, Vanderbilt \\ University School of Medicine, Nashville, TN, USA
}

\begin{abstract}
Chronic alcohol exposure can cause dramatic behavioral alterations, including increased anxiety-like behavior and depression. These alterations are proposed to be due in part to adaptations in the brain regions that regulate emotional behavior, including the bed nucleus of the stria terminalis (BNST), a principal output nucleus of the amygdala. However, to date there have been no studies that have examined the impact of in vivo alcohol exposure on synaptic function in the BNST. To better understand how alcohol can alter neuronal function, we examined the ability of in vivo alcohol exposure to alter glutamatergic transmission in the BNST using whole-cell voltage clamp recordings and biochemistry in brain slices obtained from C57BI6 mice. Chronic intermittent, but not continuous, ethanol vapor exposure increased temporal summation of NMDA receptor (NMDAR)-mediated excitatory postsynaptic currents (EPSCs). Both electrophysiological and biochemical approaches suggest that this difference is not because of an alteration in glutamate release, but rather an increase in the levels of NR2Bcontaining NMDARs. Further, we found that ethanol modulation of NMDAR in the vBNST is altered after intermittent alcohol exposure. Our results support the hypothesis that NMDAR-mediated synaptic transmission is sensitized at key synapses in the extended amygdala and thus may be a suitable target for manipulation of the behavioral deficits associated with acute withdrawal from chronic alcohol exposure. Neuropsychopharmacology (2009) 34, 2420-2429; doi:I 0.1038/npp.2009.69; published online 24 June 2009
\end{abstract}

Keywords: anxiety; withdrawal; plasticity; addiction; stress; postsynaptic

\section{INTRODUCTION}

Alcohol alters behavior through interactions with a variety of protein targets in the central nervous system, including both voltage-gated and ligand-gated ion channels. The NMDA receptor (NMDAR) is a well-characterized target of ethanol that has been associated with both drinking behavior (Wang et al, 2007) and withdrawal (Hoffman and Tabakoff, 1994). The NMDAR is a ligand-gated ion channel composed of two obligatory NR1 subunits and two NR2 or NR3 subunits that confer unique biophysical properties (see Yashiro and Philpot, 2008 for review). Acute application of alcohol to brain slices inhibits NMDAR function (Chu et al, 1995; Mirshahi and Woodward, 1995), likely through a direct interaction (Lovinger et al, 1989). This inhibition exhibits a degree of subunit selectivity in vivo, targeting either NR2A (Suvarna et al, 2005) or NR2B

*Correspondence: Dr DG Winder, Department of Molecular Physiology and Biophysics, Vanderbilt University School of Medicine, 23rd and Pierce Ave S, Room 724B, RRB, Nashville, TN 37232-0615, USA,

Tel: + | 615322 ||44, Fax: + | 615322 |462,

E-mail: Danny.winder@vanderbilt.edu

${ }^{4}$ Current address: Dr TL Kash, Department of Pharmacology, Bowles Center for Alcohol Studies, University of North Carolina Chapel Hill, NC, USA

Received 16 February 2009; revised 14 April 2009; accepted 21 May 2009
(Roberto et al, 2004; Kash et al, 2008)-containing NMDARs, depending on the region of the brain being studied. In contrast to the acute effects of alcohol, chronic alcohol exposure has been shown to lead to an upregulation of NMDAR function (Kalluri et al, 1998; Carpenter-Hyland et al, 2004; Carpenter-Hyland and Chandler, 2006). This increase in NMDAR function has been posited to play a role in the hyperexcitable state (Hendricson et al, 2007) and altered anxiety-like behavior (Gatch et al, 1999; Kiefer et al, 2003) that is associated with alcohol withdrawal, however a detailed analysis of the effects of chronic alcohol exposure on the circuitry that underlies these specific effects has not yet been performed.

The central extended amygdala, in particular the bed nucleus of the stria terminalis (BNST), has been proposed to be a critical site of action for adaptations associated with alcohol abuse (see Koob, 2008 for review). However, to date there have been no studies that have examined the impact of in vivo alcohol exposure on synaptic function in the BNST. This area of investigation is of particular interest, as the BNST has been proposed to be critical for the behavioral response to sustained fear through control of brain regions that mediate specific aspects of anxiety-like behavior (see Walker and Davis, 2008 for review). Given the proposed role that withdrawal-induced anxiety plays in regulating subsequent alcohol intake (Valdez et al, 2002; Breese et al, 2004; Funk et al, 2006), we performed experiments to understand 
how withdrawal from chronic ethanol exposure can alter neuronal function. Specifically, we examined the ability of in vivo alcohol vapor exposure to alter NMDAR function in the BNST using whole-cell voltage clamp recordings in brain slices. We focused on the vBNST because this region has been shown to regulate activation of both stress (Spencer et al, 2005) and reward pathways (Georges and Aston-Jones, 2002). In addition, we had previously performed an extensive ex vivo characterization of NMDARmediated synaptic transmission and acute modulation by ethanol in the vBNST (Kash et al, 2008), providing us the framework to build an integrated model of both the acute actions of alcohol on NMDAR and the ability of chronic alcohol exposure to regulate NMDAR function. Here, we report that during withdrawal from chronic intermittent alcohol vapor exposure, there is an increase in the temporal summation of NMDAR-mediated synaptic transmission in the vBNST. This alteration in temporal summation is not because of alterations in glutamate release, but rather an increase in decay time due in part to increased contribution of NR2B-containing NMDARS to synaptic transmission. On the basis of our earlier findings we propose a model in which homeostatic regulation of the NR2B subunit plays a key role in both acute ethanol inhibition of NMDARs and chronic alcohol withdrawal-induced effects on glutamatergic synaptic function in the BNST.

\section{METHODS}

\section{Chronic Ethanol Procedure}

All procedures were performed according to the Vanderbilt University Institutional Animal Care and Use Committee approved procedures. Procedures were performed as outlined in the INIA-Stress standard operating procedure (www.iniastress.org) and as performed earlier in the Winder Lab (Healey et al, 2008). Briefly, male C57Bl/6J mice (6-7-weeks old, Jackson Laboratories) were given a daily injection of either pyrazole (control group, control, $1 \mathrm{mmol} /$ $\mathrm{kg}$ ) or pyrazole + ethanol (ethanol group, EtOH, $1 \mathrm{mmol} / \mathrm{kg}$ $+0.8 \mathrm{~g} / \mathrm{kg}$, respectively) to impair the metabolism of ethanol. Thirty minutes after the injection, in their home cages, mice were placed in a chamber filled with volatilized ethanol (EtOH, 20.3 $\pm 0.2 \mathrm{mg} / \mathrm{l}$ ) or volatilized water (control). Airflow through the chambers was maintained at $5.5 \mathrm{l} / \mathrm{min}$ and volatilization at $1.5 \mathrm{l} / \mathrm{min}$. Mice were allowed food and water ad libitum. After $16 \mathrm{~h}$ of exposure, mice were removed from the chambers and placed in fresh cages. Using these parameters we can reliably obtain blood ethanol concentrations in the range of $150-185 \mathrm{mg} / \mathrm{dl}$ (Healey et al, 2008). This corresponds to a blood alcohol level of $0.21 \%$. For the chronic intermittent ethanol exposure paradigm, four $16 \mathrm{~h}$ exposures occurred with four $8 \mathrm{~h}$ withdrawals after each exposure. For the chronic continuous ethanol exposure paradigm, mice were exposed for a single $64 \mathrm{~h}$ session with daily injections of pyrazole.

\section{Elevated Plus Maze and Behavioral Analysis}

The elevated plus maze (EPM) follows the general design described by Lister (1987). The EPM comprises two closed arms $\left(30 \times 10 \times 5 \mathrm{~cm}^{3}\right)$ and two open arms $\left(30 \times 10 \times 5 \mathrm{~cm}^{3}\right)$ that meet at the junction $\left(5 \times 5 \mathrm{~cm}^{2}\right)$ of the four arms. The floor of the EPM was made of clear plexiglass. The walls of the enclosed arms were $20 \mathrm{~cm}$ high and made of black plexiglass. A small $(0.25 \mathrm{~cm})$ edge provided grip for the animals in the open arms. The entire EPM apparatus was elevated $50 \mathrm{~cm}$ above floor level. The lights of the laboratory were dimmed (a $40 \mathrm{~W}$ bulb was placed behind a solid screen to allow for diffuse lighting). To insure approximately equal light distribution, Lux levels were assessed mid-length along each of the four arms and at the junction of the EPM. One hour before testing, the mice were brought to the EPM testing laboratory. For each mouse, the EPM testing was conducted between 4 and $6 \mathrm{~h}$ after the final vapor chamber exposure. Testing began by placing the subject on the central platform (facing an open arm). The videotaped test sessions were $5 \mathrm{~min}$ in duration. Between test sessions, the maze was thoroughly cleaned with $20 \%$ ethanol. Videotapes were automatically scored with NIH Image software for open and closed arm entries, time spent in the open and closed arms and total distance traveled.

\section{Brain Slice Preparation}

Male C57Bl/6J mice (6-8-weeks old, Jackson Laboratories) were decapitated under anesthesia (Isoflurane). The brains were quickly removed and placed in ice-cold sucroseartificial cerebrospinal fluid (ACSF): (in mM) 194 sucrose, $20 \mathrm{NaCl}, 4.4 \mathrm{KCl}, 2 \mathrm{CaCl}_{2}, 1 \mathrm{MgCl}_{2}, 1.2 \mathrm{NaH}_{2} \mathrm{PO}_{4}, 10.0$ glucose, and $26.0 \mathrm{NaHCO}_{3}$ saturated with $95 \% \mathrm{O}_{2} / 5 \% \mathrm{CO}_{2}$. Slices of $300 \mu \mathrm{m}$ in thickness were prepared using a Tissue Slicer (Leica). Slices were then stored in a heated (approximately $\left.28^{\circ} \mathrm{C}\right)$, oxygenated $\left(95 \% \quad \mathrm{O}_{2}-5 \% \quad \mathrm{CO}_{2}\right)$ holding chamber containing 'normal' ACSF [ACSF: (in mм) $124 \mathrm{NaCl}, 4.4 \mathrm{KCl}, 2 \mathrm{CaCl}_{2}, 1.2 \mathrm{MgSO}_{4}, 1 \mathrm{NaH}_{2} \mathrm{PO}_{4}, 10.0$ glucose, and 26.0 NaHCO3] or transferred to a submerged recording chamber where they were perfused with heated (28-30 , unless otherwise noted), oxygenated ACSF at a rate of about $2 \mathrm{ml} / \mathrm{min}$. Slices were allowed to equilibrate in normal ACSF for $1 \mathrm{~h}$ before experiments began.

\section{Whole-Cell Voltage Clamp Recordings}

Slices were placed in a submerged chamber (Warner Instruments) and neurons of the vBNST were directly visualized with infrared video microscopy (Olympus). Recording electrodes (3-6 M 2 ) were pulled on a FlamingBrown Micropipette Puller (Sutter Instruments) using thinwalled borosilicate glass capillaries. NMDA-excitatory postsynaptic currents (EPSCs) were evoked by local fiber stimulation with bipolar ni-chrome electrodes. Stimulating electrodes were placed in the vBNST, $100-500 \mu \mathrm{m}$ medial from the recorded neuron, and electrical stimuli $(5-40 \mathrm{~V}$ with a $100-150 \mu$ s duration) was applied at $0.2 \mathrm{~Hz}$ unless otherwise noted. NMDA EPSCs were pharmacologically isolated by adding $25 \mu \mathrm{M}$ picrotoxin and $10 \mu \mathrm{M}$ NBQX and recording at a holding potential of $+40 \mathrm{mV}$. Recording electrodes for were filled with (in $\mathrm{mM}$ ) $\mathrm{Cs}^{+}$-gluconate (135), $\mathrm{NaCl}$ (5), HEPES (10), EGTA (0.6), ATP (4), GTP (0.4), pH $7.2,290-295 \mathrm{mOsmol}$. Signals were acquired through a Multiclamp 700B amplifier (Axon Instruments), digitized 
and analyzed through pClamp 9.2 software (Axon Instruments).

Input resistance, holding current, and series resistance were all monitored continuously throughout the duration of experiments. Experiments in which changes in series resistance were greater than $20 \%$ were not included in the data analysis. Experiments were analyzed by measuring the parameter of interest, peak amplitude, or area of the synaptic response, which was normalized to the baseline period. The baseline period is defined as the $5 \mathrm{~min}$ period immediately preceding application of the drug. NMDA EPSC decay was fitted with two exponentials using Clampfit 9.2 for averages of several traces from baseline and 'drug' values as done earlier. To allow for direct comparison of decay times between experimental conditions, the two decay time components, $\tau_{1}$ and $\tau_{2}$, were combined into a weighted time constant, $\tau_{\mathrm{w}}$, using the equation: $\tau_{\mathrm{w}}=\left(\tau_{1}^{\star} a_{1}\right)+\left(\tau_{2}^{\star} a_{2}\right)$, where $a_{1}$ and $a_{2}$ are the relative amplitudes of the two exponential components. AMPA EPSC decay was fitted using a single exponential in Clampfit 9.2. Statistical analyses were performed using Microsoft Excel, Graphpad Prism, and Microcal Origin. To determine significance between alcohol-exposed and control electrophysiological parameters, two-tailed unpaired Student's $t$-tests were used, unless otherwise noted. AMPA-mediated sEPSCs were recorded from a holding potential of $-70 \mathrm{mV}$ and pharmacologically isolated by adding $25 \mu \mathrm{M}$ picrotoxin to ACSF. SEPSC recordings were acquired in $2 \mathrm{~min}$ gap-free blocks.

\section{Pharmacology}

Picrotoxin, Ro 25-6981, and DL-2-Amino-5-phosphonopentanoic acid (DL-APV) were purchased from Sigma-Aldrich (St Louis, MO). NBQX was purchased from Ascent (United Kingdom). Ethanol (95\%) was purchased from Aaper Alcohol and Chemical (Shelbyville, KY). DMSO (0.05\%) was used as a vehicle for picrotoxin.

\section{Western Blotting}

Tissue punches enriched in the vBNST were obtained from freshly prepared $400 \mu \mathrm{M}$ brain slices as described earlier (Healey et al, 2008). Samples were sonicated in $150 \mu \mathrm{l}$ of a $2 \%$ SDS containing $10 \mu \mathrm{g} / \mathrm{ml}$ leupeptin and $1 \mu \mathrm{g} / \mathrm{ml}$ pepstatin. After sonication, total protein levels were measured for each sample using BCA assay (Thermo Fisher Scientific). Proteins (3-5 $\mu \mathrm{g}$ total) were separated by SDS-PAGE (10\%), and then transferred to nitrocellulose membrane. Membranes were stained with PonceauS solution and scanned before being blocked in two changes of 5\% non-fat milk in $50 \mathrm{mM}$ Tris- $\mathrm{HCl} \mathrm{pH} 7.5,0.15 \mathrm{M} \mathrm{NaCl}, 0.05 \%$ Tween-20 (TBST) for $30 \mathrm{~min}$ each. Membranes were next incubated with either NR1 (Mouse, BD Transduction), NR2A (Rabbit, Millipore), NR2B (Mouse, BD Transduction) GluR1 (Mouse, AbCam), or CaMKII (Goat; McNeill and Colbran, 1995) antibodies overnight. Membranes were washed with five changes of 5\% non-fat milk made in TBST, then incubated with the appropriate secondary antibody for $1 \mathrm{~h}$. Membranes were then washed in five changes of TBST, briefly washed in $\mathrm{ddH} 2 \mathrm{O}$, and then Western Lightning Plus chemiluminescence reagent (Perkin Elmer). Blots were

exposed to X-ray film (Phenix) and band intensity was quantified using NIH Image J. Band intensity was normalized to the total protein loaded as determined from the intensity of PonceauS stain. Data points from control and ethanol treated samples were normalized to the average of the controls within each set of blots: mean \pm SEM values are reported and were compared using an unpaired $t$-test.

\section{RESULTS}

\section{Performance in the EPM is Impaired in C57bl6j Mice During Acute Withdrawal from Chronic Intermittent Alcohol Vapor Exposure}

Chronic intermittent ethanol vapor exposure has been shown to have numerous effects, including increased alcohol drinking behavior (Griffin et al, 2009), handlinginduced convulsions (Becker et al, 1997), and anxiety (Kliethermes et al, 2004). The performance of mice during acute withdrawal (4-6h after removal) from intermittent alcohol or air exposure was evaluated in the EPM to demonstrate that the animals that underwent chronic intermittent alcohol vapor exposure have altered behavior consistent with alcohol withdrawal (Figure 1). We focused on this time-point, as earlier studies have shown that there is a significant increase in handling-induced convulsion (Becker et al, 1997), suggesting a hyperexcitable state in the CNS. We found that, at this time-point, mice exposed to intermittent alcohol $(n=5)$ traveled significantly less total distance than air-exposed mice $(n=8)$ (Figure 1a, $\left.t(11)=3.56,{ }^{*} p<0.05\right)$, consistent with a recent report that motor impairment is a withdrawal phenotype in mice (Philibin et al, 2008). Further, alcohol-exposed mice had a

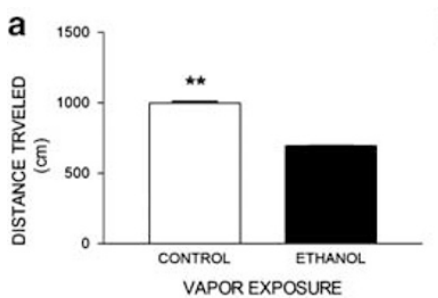

C

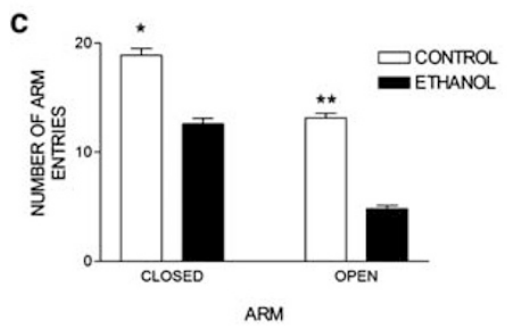

Figure I Anxiety-related behavior as measured in the 5 min duration elevated plus maze (EPM) test was assessed 4-6h after the last chronic intermittent ethanol or air vapor (control) exposure. (a) Total distance traveled during the 5 min test session. (b) Time (s) spent in the closed and open arm of the EPM. (c) Number of open and closed arm entries on the EPM. (d) Schematic diagram of illumination levels during the EPM test (numerical values in $L x$ ). Each value represents the mean \pm SEM. Control $(n=8)$, ethanol $(n=5)$. *P<0.05 (control group compared with ethanol vapor-exposed group), $* * P<0.0$ I (control group compared with ethanol vapor-exposed group), unpaired Student's two-tailed t-test. 
significant reduction in time spent in the open arm and open arm entries, consistent with an increase in anxiety-like behavior (Figure $1 \mathrm{~b}$ and c), respectively, $t(11)=3.76$, $\left.{ }^{*} p<0.05\right)$.

\section{Acute Withdrawal from Chronic Intermittent, but not Chronic Continuous, Alcohol Vapor Exposure Enhances the Temporal Summation of NMDAR EPSCS in the vBNST}

Given the postulated critical role of the extended amygdala in alcohol abuse, in particular the negative affect associated with withdrawal, we examined NMDAR function in the vBNST during acute withdrawal from an intermittent alcohol exposure. NMDAR function in other brain regions can be regulated by alcohol exposure; but to date there has been no examination of the impact of chronic ethanol exposure on NMDAR function in the vBNST. We focused on temporal summation of NMDAR, as this has been suggested to be a marker for metaplasticity, or the plasticity of synaptic plasticity, (Philpot et al, 2007) and is sensitive to alterations both in synaptic and extrasynaptic NMDAR function and glutamate release. We pharmacologically isolated NMDAR EPSCs using NBQX and picrotoxin and then examined the response to bursts of stimulation (10 pulses) at 10,20, and $40 \mathrm{~Hz}$. We found that in vBNST neurons from mice exposed to intermittent alcohol $(n=13)$ there was a significant increase in temporal summation of NMDAR EPSCs at 10 and $20 \mathrm{~Hz}$ but not $40 \mathrm{~Hz}$ (Figure 2) when compared with the corresponding controls $(n=10)$ (amplitude of the 10th pulse/1st pulse: $10 \mathrm{~Hz}$ : control: $1.30 \pm 0.10$; intermittent alcohol: $1.91 \pm 0.18 ; t(21)=2.84$, $p<0.01 ; 20 \mathrm{~Hz}$ : control: $1.50 \pm 0.15$; intermittent alcohol: $2.08 \pm 0.21 ; t(21)=2.33 p<0.01 ; 40 \mathrm{~Hz}$ : control: $2.05 \pm 0.20$; intermittent alcohol: $2.52 \pm 0.21 ; t(21)=1.68, p=0.10$ ). When we examined the area under the normalized temporal summation curve (a value that we termed the summation index and is similar to the normalized total charge transfer) there is a significant effect $(p<0.05)$ at both 10 and $20 \mathrm{~Hz}$, with a strong trend for significance at $40 \mathrm{~Hz}(p=0.09)$ Figure 2e).

We were next interested if this alteration in temporal summation of NMDAR EPSCs was due to alcohol exposure, or perhaps some combination of alcohol exposure and withdrawal. To investigate this possibility we used a chronic continuous alcohol vapor paradigm in which mice were exposed to alcohol for the same total time $(64 \mathrm{~h})$ but with only one withdrawal. We found that there was no alteration in the temporal summation of NMDAR EPSCs in the vBNST after this continuous alcohol exposure $(n=11)$ when compared with the corresponding controls $(n=7)$ (amplitude of the 10th pulse/1st pulse: $10 \mathrm{~Hz}$ : control: $1.35 \pm 0.20$; continuous alcohol: $1.50 \pm 0.17 ; t(16)=0.63, p=0.54 ; 20 \mathrm{~Hz}$ : control: $\quad 1.41 \pm 0.16$; continuous alcohol: $1.76 \pm 0.14$; $t(16)=0.34, p=0.74 ; 40 \mathrm{~Hz}$ : control:1.80 \pm 0.20 ; continuous alcohol: $2.20 \pm 0.19 ; t(16)=0.08, p=0.94)$. This lack of an effect is clearly shown when the summation index is plotted for these experiments (Figure 2f). These results suggest that the alteration of temporal summation observed after intermittent alcohol exposure is mediated by both alcohol exposure and the multiple withdrawals. We next investigated the persistence of this alteration of synaptic function. a
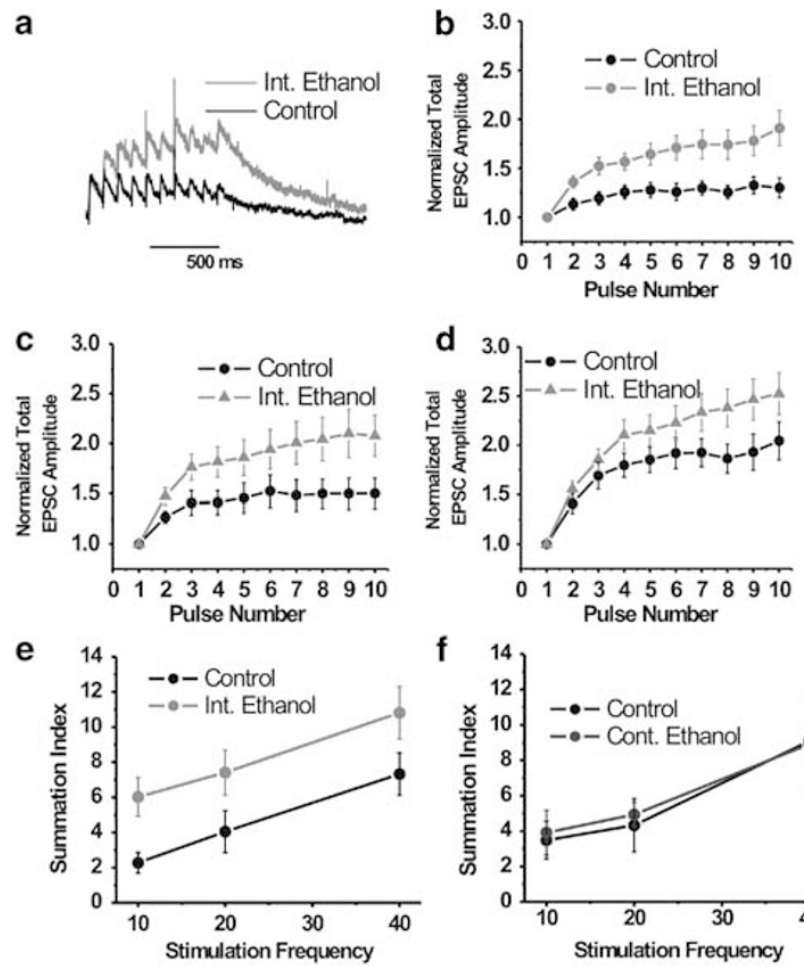

f

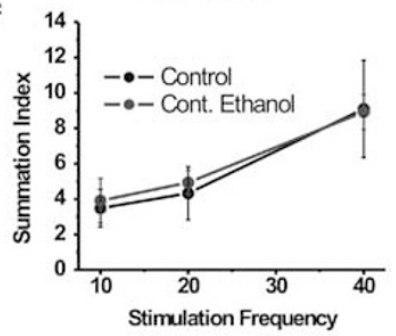

Figure 2 Temporal summation of NMDAR EPSCs in the $V B N S T$ is enhanced after intermittent but not continuous alcohol exposure. (a) Representative normalized trace demonstrating the ability of 10 stimulation pulses given at $10 \mathrm{~Hz}$ to induce enhanced temporal summation of NMDAR EPSCs after intermittent alcohol exposure. (b) Pooled data demonstrating the increase in temporal summation of NMDAR EPSCs at a stimulation frequency of $10 \mathrm{~Hz}$ after intermittent alcohol exposure. (c) Pooled data demonstrating the increase in temporal summation of NMDAR EPSCs at a stimulation frequency of $20 \mathrm{~Hz}$ after intermittent alcohol exposure. (d) Pooled data demonstrating the increase in temporal summation of NMDAR EPSCs at a stimulation frequency of $40 \mathrm{~Hz}$ after intermittent alcohol exposure. (e) Plot of the summation index across different stimulation frequencies and treatments demonstrating the significant increase in temporal summation after intermittent alcohol exposure. (f) Plot of the summation index across different stimulation frequencies and treatments demonstrating the lack of a significant increase in temporal summation after continuous alcohol exposure.

We found that 1 week after removal from alcohol exposure there was no alteration in temporal summation of NMDAR EPSCs in the vBNST (data not shown). This finding suggests that this intermittent alcohol-induced alteration of NMDAR function in the vBNST is a transient event associated with acute withdrawal.

Acute Withdrawal from Intermittent Alcohol Exposure does not Alter the Probability of Glutamate Release in the vBNST

We next sought to determine the mechanism underlying the alteration in temporal summation of NMDAR EPSCs in the vBNST during acute withdrawal from intermittent alcohol exposure. One potential mechanism underlying this enhancement could be through an increase in glutamate release (Yashiro et al, 2005). To evaluate this possibility, we assessed glutamate release using multiple complementary techniques. We first examined spontaneous AMPAR- 
mediated glutamatergic synaptic transmission (sEPSCs) (Figure 3a). This technique is useful in such that if there were a large increase in synaptic glutamate release one would see an increase in the frequency of sEPSCs. We found that there was no significant difference in either the frequency (Figure $3 b ; p=0.36$ ) or the amplitude (Figure $3 c$; $p=1)$ of AMPAR-mediated sEPSCs in mice exposed to intermittent alcohol $(n=10)$ when compared with control $(n=9)$ mice. We next examined the paired-pulse ratio (PPR) of evoked AMPAR-mediated EPSCs (Figure 3d). If there were an increase in the probability of glutamate release, one would expect a corresponding decrease in the PPR. Consistent with the lack of an alteration of sEPSC frequency, we found no significant alteration in the PPR at a variety of interstimulus intervals in mice exposed to intermittent alcohol $(n=12)$ when compared with controls $(n=12)$ (Figure 3e). As a final means of investigating glutamate release after intermittent alcohol exposure, we examined the rate of blockade of the activity-dependent NMDAR antagonist MK-801 and found no significant differences from the control experiments (MK-801 blocking decay time in control mice $(n=3): 37 \pm 4 \mathrm{~s}$, intermittent alcohol mice $(n=3): 49 \pm 14 \mathrm{~s} p=0.49)$. In combination, these results suggest that the increase in temporal summation of NMDAR EPSCs in the vBNST during acute
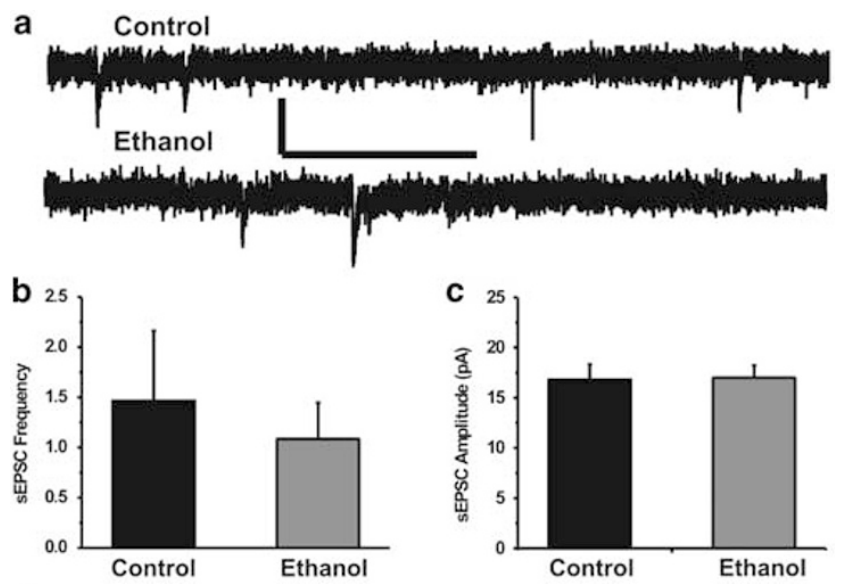

d

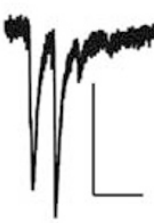

Control

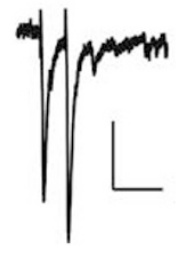

Ethanol

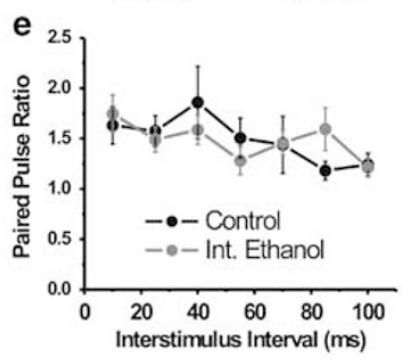

Figure 3 Acute withdrawal from intermittent alcohol exposure does not alter glutamate release in the VBNST. (a) Representative sEPSC traces from control and intermittent alcohol exposed mice demonstrating the lack of an effect of intermittent alcohol exposure on spontaneous glutamatergic transmission. Scale bars represent $25 \mathrm{pA}$ and $250 \mathrm{~ms}$ respectively. (b) Intermittent alcohol exposure does not alter the SEPSC frequency. (c) Intermittent alcohol exposure does not alter sEPSC amplitude. (d) Representative traces of evoked AMPA EPSCs demonstrating the lack of an effect of intermittent alcohol treatment on the paired pulse ratio. Scale bars represent 100 pA and 100 ms respectively. (e) Pooled data demonstrating the lack of an effect of intermittent alcohol exposure on the paired pulse ratio across a range of interstimulus intervals. withdrawal from intermittent alcohol exposure is likely not because of an alteration in glutamate release.

Acute Withdrawal from Intermittent Alcohol Exposure Leads to Increased Decay Kinetics of Synaptic NMDAR-Mediated Currents in the vBNST

Having established that there is no alteration in glutamate release in the vBNST during acute withdrawal from intermittent alcohol exposure we next focused our efforts on examining the properties of synaptic NMDARs. One means by which temporal summation of NMDAR EPSCs could be altered is an alteration of the NMDAR decay kinetics (Philpot et al, 2007). To evaluate this, we examined the kinetics of NMDAR EPSCs in response to a single stimulus. We found that after the intermittent alcohol exposure paradigm, there was a significant increase in the decay time of NMDAR EPSC in vBNST neurons when compared with the control animals (Figure $4 \mathrm{a}$ and $\mathrm{b}$ ) (control, $n=14$, weighted $\tau$ : $125 \pm 16 \mathrm{~ms}$; intermittent alcohol, $n=17$. weighted $\tau: 201 \pm 25 \mathrm{~ms}, \quad t(29)=2.80$, $\left.{ }^{*} p<0.01\right)$. To determine if this alteration in decay time was specific to NMDA-mediated transmission, we examined the decay time of evoked AMPA EPSCs recorded at $-70 \mathrm{mV}$. We found that though there was no significant difference, there was a strong trend toward a decrease in the decay time of AMPA EPSCs in vBNST neurons from control mice $(9.1 \pm 1.8 \mathrm{~ms}, \quad n=8)$ when compared with intermittent alcohol-exposed mice $(6.1 \pm 0.8 \mathrm{~ms}, n=12, t(18)=1.79$, $p=0.09$ ).

\section{Intermittent Alcohol Exposure Increases the Functional Expression of NR2B-Containing Synaptic NMDAR in the vBNST}

We next sought to determine the mechanism underlying the intermittent alcohol-induced alteration in NMDAR EPSC decay kinetics. One potential reason for this alteration in NMDAR-mediated synaptic transmission could be an alteration in the tonic excitability of the neurons. To evaluate this, we examined the membrane resistance of these neurons at both $-70 \mathrm{mV}$ (control: $478 \pm 45 \mathrm{M} \Omega$, $n=18$; intermittent alcohol: $501 \pm 51 \mathrm{M} \Omega, n=18, p=0.71$ )
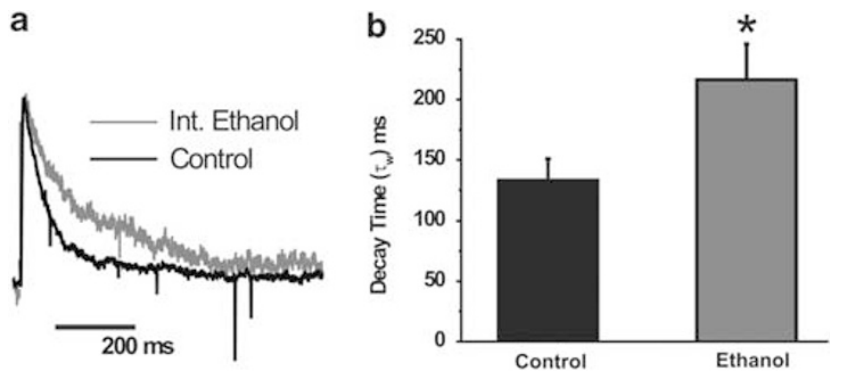

Figure 4 Acute withdrawal from intermittent alcohol exposure alters NMDAR kinetics in the vBNST. (a) Representative normalized NMDAR EPSC traces from mice exposed to intermittent alcohol and corresponding controls demonstrating the lengthening of the decay kinetics. (b) Pooled results demonstrating that intermittent alcohol exposure (red) leads to a significant increase in the decay time as denoted by the weighted $\tau$. ${ }^{*} p<0.05$, unpaired Student's two-tailed $t$-test. The color reproduction of this figure is available on the html full text version of the paper. 
and $+40 \mathrm{mV}$ (control: $298 \pm 42 \mathrm{M} \Omega, n=10$; intermittent alcohol: $211 \pm 48 \mathrm{M} \Omega, n=10, p=0.32$ ) and found no significant difference. This suggests that there are no gross alterations in the passive membrane properties of vBNST neurons during acute withdrawal from chronic intermittent alcohol exposure. Another means by which NMDAR decay kinetics can be regulated is by altering the subunit content of the NMDARs. Specifically, several studies have shown that increased incorporation of NR2B into synaptic NMDARs can lead to a lengthening of decay time (Philpot et al, 2001) due in part to the biophysical properties conferred by the presence of NR2B (Erreger et al, 2005). We investigated the functional contribution of NR2B-containing synaptic NMDAR in the vBNST after intermittent alcohol exposure by examining the effect of the NR2Bselective antagonist, Ro 25-6981. Similar to earlier studies from naïve mice from our laboratory (Kash et al, 2008), we found that bath application of Ro 25-6981 inhibited NMDAR EPSCs in control mice with no significant alteration in decay kinetics $(n=5$, Figure 5a, c-e, $p=0.74)$. In contrast, we found a modest increase in the
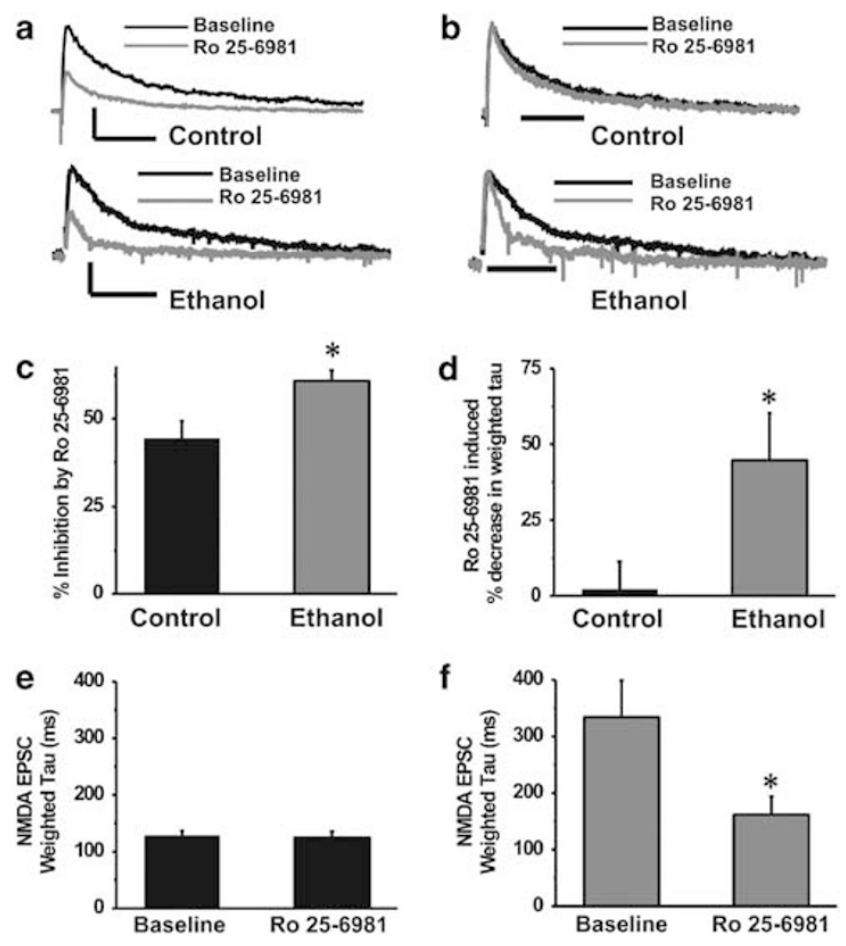

Figure 5 Acute withdrawal from intermittent alcohol exposure leads to an upregulation of NR2B in the vBNST. (a) Representative NMDAR EPSC traces showing the effect of Ro 25-698 I in control and intermittent alcoholexposed mice. Scale bars represent 50 pA and 200 ms respectively. (b) Representative normalized NMDAR EPSC traces showing the altered effect of Ro 25-698I on decay kinetics following intermittent alcohol exposure. Scale bar represents $200 \mathrm{~ms}$ respectively. (c) Pooled data demonstrating the increased Ro 25-698I inhibition of NMDAR EPSC area after intermittent alcohol exposure. $* 0<0.05$, unpaired Student's two-tailed t-test. (d) Pooled data demonstrating the alteration in the ability of Ro 25-698I to modulate the decay kinetics of the NMDAR EPSC after intermittent alcohol exposure. ${ }^{*} p<0.05$, unpaired Student's two-tailed t-test. (e) Pooled data demonstrating the effect of Ro 25-698I on decay kinetics of NMDAR EPSCs in control mice. (f) Pooled data demonstrating the effect of Ro 25-698I on decay kinetics of NMDAR EPSCs in mice exposed to intermittent alcohol. ${ }^{*} p<0.05$, paired Student's one-tailed t-test. ability of Ro 25-6981 to inhibit NMDAR EPSCs in mice that were exposed to intermittent alcohol (control, $n=5$, percent reduction in NMDAR EPSC area compared with baseline: $44 \pm 5$; intermittent alcohol, $n=4$, percent reduction in NMDAR EPSC area compared with baseline: $61 \pm 3$, $t(7)=2.50, p<0.05$ ) (Figure $5 \mathrm{~b}$ and $c$ ). More importantly, in mice exposed to intermittent alcohol there was an increase in the ability of Ro 25-6981 to alter decay kinetics when compared with control mice (control, $n=5$, percent change in NMDAR EPSC weighted $\tau$ compared with baseline: $-2 \pm 9$; intermittent alcohol, $n=4$, percent change in NMDAR EPSC weighted $\tau$ compared with baseline: $-49 \pm 13 \%, t(7)=4.00,{ }^{*} p<0.01$ ) (Figure $5 \mathrm{~d}$ and $\mathrm{f}$ ). This result supports the possibility that there is an increase in the contribution of NR2B to synaptic NMDARs in the vBNST after intermittent alcohol exposure. Taken together, these results suggest a shift in synaptic NMDAR from triheteromeric (NR1/NR2A/NR2B) NMDARs to a population more enriched in diheteromeric (NR1/NR2B) NMDARs.

\section{Acute withdrawal from Intermittent Alcohol Exposure Alters Protein Expression of NMDAR NR2B Subunits and CaMKII $\alpha$ in the vBNST}

The alteration of NMDA-mediated synaptic transmission in the vBNST after alcohol exposure could be because of an alteration in the expression levels of NR2B. To test this possibility, we evaluated the expression levels of NR1, $\mathrm{NR} 2 \mathrm{~A}$, and NR2B in tissue punches enriched in the vBNST from intermittent alcohol-exposed and control mice (Figure 6). We found that NR2B expression was significantly increased approximately 1.7 -fold in intermittent alcohol-exposed mice $(t(33)=2.865, p<0.01)$, whereas NR1 $(p=0.61)$ and NR2A $(p=0.63)$ expression was unchanged. These results strongly support the possibility that the alteration in synaptic NMDAR function properties is due to increased levels of NR2B in the vBNST. We then compared the expression levels of the AMPA receptor subunit, GluR1, and found no differences in between intermittent alcohol exposed and control groups $(p=0.69)$. We next compared the expression levels of calcium/calmodulin-dependent protein kinase II (CaMKII $\alpha$ ) in control and intermittent alcohol-exposed mice. This protein is of particular interest because it interacts with and phosphorylates NR2B (Strack et al, 2000) and also modulates NMDAR channel kinetics (Sikes et al, 2005). Moreover, CaMKII binding to NR2B is critical for the induction of plasticity (Barria and Malinow, 2005). Intermittent alcohol exposure significantly reduced the expression of CaMKII $\alpha$ in the vBNST by approximately $19 \%(t(16)=2.240, p<0.05)$.

\section{Acute Withdrawal from Intermittent Alcohol Exposure Leads to Altered Acute Ethanol Sensitivity of Synaptic NMDAR-Mediated Currents in the vBNST}

We have shown earlier that alcohol inhibits NMDAR EPSCs in the vBNST in an NR2B-dependent fashion (Kash et al, 2008). Specifically, we found that in the presence of the NR2B-selective antagonist, Ro 25-6981, $50 \mathrm{mM}$ ethanol did not further inhibit NMDA EPSCs. On the basis of this, we 
a

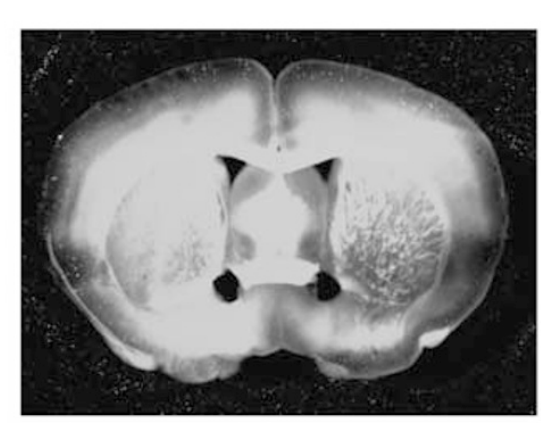

b

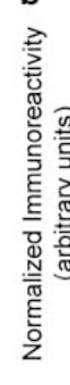

NR1

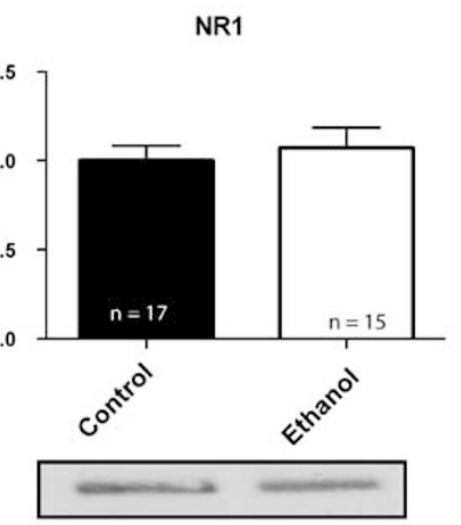

C

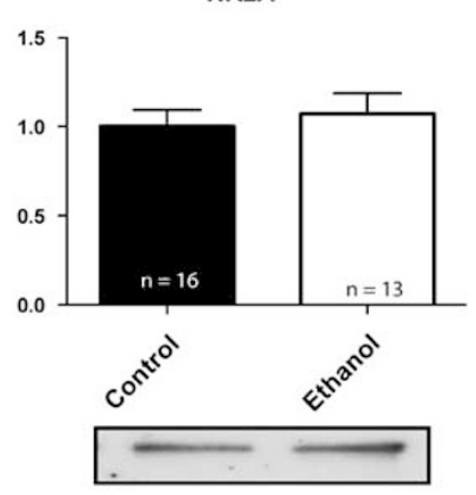

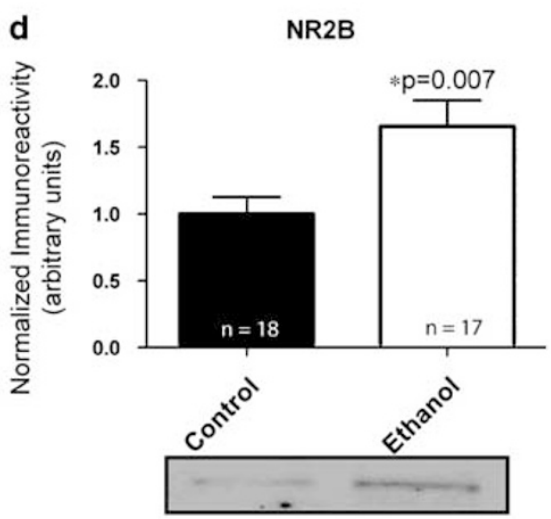

e

GluR1

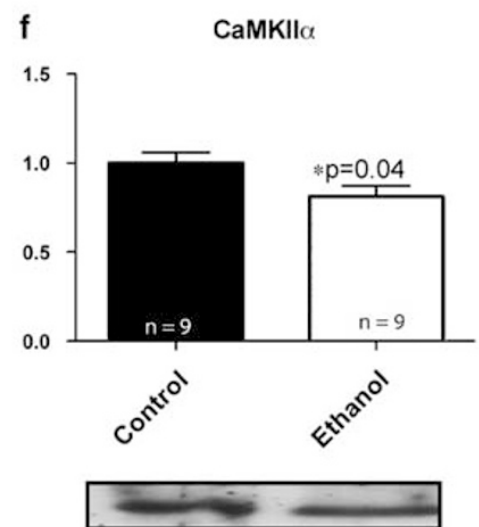

Figure 6 Acute withdrawal from intermittent alcohol exposure leads to an upregulation of levels of NR2B protein and a downregulation of CaMKIl $\alpha$ in the vBNST. (a) Representative brain slice demonstrating the location and size of the vBNST punches. (b) There is no difference in NRI protein expression

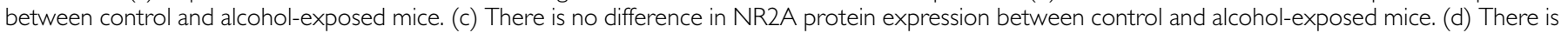
a significant increase in NR2B protein expression in alcohol-exposed mice compared with control mice. * $p<0.05$, unpaired Student's two-tailed $t$-test.

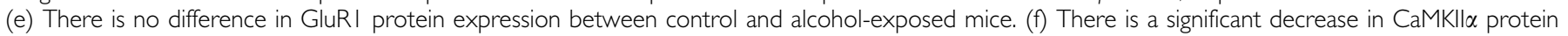
expression in alcohol-exposed mice compared with control mice. * $p<0.05$,unpaired Student's two-tailed t-test.

reasoned that after intermittent alcohol exposure there would be an increase in the ability of ethanol to inhibit NDMA EPSCs in the vBNST. Unexpectedly, we found that there was a reduction in the ability of $50 \mathrm{mM}$ ethanol to inhibit peak amplitude of NMDA EPSCs after intermittent alcohol exposure (control, $n=5,43 \pm 11 \%$ reduction in peak amplitude compared with baseline; alcohol, $n=5$, $15 \pm 5 \%$ reduction in peak amplitude compared with baseline; $t(8)=2.48, p<0.05$, Figure $7 \mathrm{a}$ ) and a slight reduction in the ability to inhibit NMDA EPSC area (control, $n=5,45 \pm 20 \%$ reduction in area compared with baseline; alcohol, $n=5,14 \pm 12 \%$ reduction in peak amplitude compared with baseline; $p=0.15$, Figure $7 b$ ). Similar to the effects of alcohol exposure on temporal summation, there was no difference in the ability of alcohol to inhibit NMDA EPSCs after continuous alcohol exposure (data not shown). One explanation for the lack of an effect on peak amplitude and a slight effect on the area was that $50 \mathrm{mM}$ ethanol was altering decay in the alcohol-exposed mice. However, there was no effect of $50 \mathrm{mM}$ ethanol on NMDA EPSC decay kinetics after intermittent alcohol exposure (Figure 7d). Consistent with earlier results obtained in naïve animals, we found no effect of $50 \mathrm{mM}$ ethanol on NMDA EPSC decay kinetics (Figure 7c).

\section{DISCUSSION}

The extended amygdala has been proposed to be involved in the negative affect associated with withdrawal from alcohol exposure (Koob, 2008). However, to date no studies have examined the ability of in vivo alcohol exposure to induce alterations in synaptic function in the BNST, one of the principal components of the extended amygdala. We show that during acute withdrawal from chronic intermittent alcohol vapor exposure, there is a reduction in total distance traveled, time spent in the open arm, and open arm entries of the EPM. These behaviors are consistent with a motor impairment (Philibin et al, 2008) and increased anxiety-like behavior (Overstreet et al, 2002), earlier described components of alcohol withdrawal. During acute withdrawal from chronic intermittent alcohol exposure we show an increase in temporal summation of NMDAR-mediated synaptic responses, in the vBNST. Our results suggest that NMDARmediated synaptic transmission is sensitized at key synapses in the extended amygdala and thus may be a suitable target for manipulation of the behavioral deficits associated with acute withdrawal from chronic alcohol exposure. 

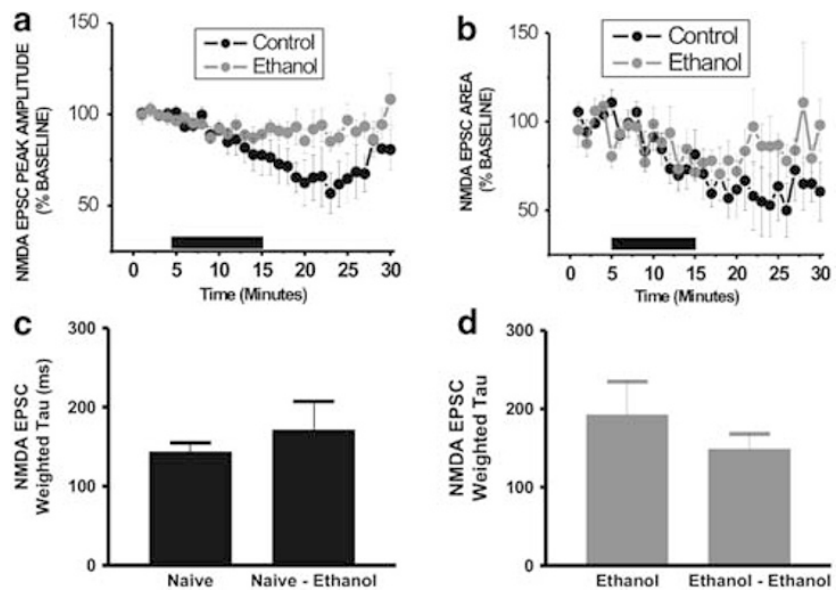

Figure 7 Ethanol sensitivity of NMDA EPSCs in the VBNST is altered during acute withdrawal from intermittent alcohol exposure. (a) The ability of bath applied $50 \mathrm{mM}$ ethanol to reduce NMDA EPSC peak amplitude is reduced in the $v B N S T$ from mice that were exposed to intermittent alcohol when compared with control mice. (b) The ability of bath applied $50 \mathrm{mM}$ ethanol to reduce NMDA EPSC area is reduced in the VBNST from mice that were exposed to intermittent alcohol when compared with control mice. (c) $50 \mathrm{mM}$ ethanol had no effect on NMDA EPSC decay kinetics in the vBNST from control mice. (d) $50 \mathrm{mM}$ ethanol had no effect on NMDA EPSC decay kinetics in the VBNST from intermittent alcoholexposed mice.

Here we show, using multiple techniques, that the alteration in temporal summation of NMDARs during acute withdrawal from intermittent alcohol vapor exposure is due to increased expression and unique pharmacological and biophysical properties of the NR2B subunit compared to the NR2A subunit. Specifically, we found an increase in the decay time of NMDAR EPSCs, but not AMPAR EPSCs, consistent with increased NR2B expression. Additionally, we found an increased sensitivity to the NR2B-selective antagonist Ro 25-6981 as reflected by a greater Ro 25-6981induced reduction in evoked NMDAR EPSCs and Ro 25-6981-induced alterations in decay kinetics. The alteration in the ability of Ro 25-6981 to alter decay kinetics, suggests an increase in the proportion of NR1/NR2B heterodimeric NMDAR. In support of these electrophysiological findings, we found that at the same time-point there was a selective increase in the expression of NR2B protein from punches containing the vBNST. Another possibility consistent with our results is that altered glutamate transport after chronic intermittent alcohol vapor exposure contributes to the enhanced temporal summation. For example, a reduction in glutamate transport would lead to increased glutamate spillover and activation of extrasynaptic NMDARs. In keeping with this possibility, chronic alcohol exposure has been shown to modulate the function of glutamate transporters in the nucleus accumbens (Melendez et al, 2005). It is critical to note that we cannot rule out the possibility that extrasynaptic NMDARs are also modified after alcohol exposure.

Curiously, we also detected a significant decrease in the total levels of CaMKII $\alpha$ after ethanol exposure. This is intriguing because increased $\mathrm{CaMKII} \alpha$ expression enhances the extent of desensitization of NR2B-containing NMDARs in heterologous cells (Sikes et al, 2005), but see Xu et al
(2008). Thus, reduced levels of CaMKII $\alpha$ in alcohol-exposed vBNST also may contribute to changes in synaptic NMDAR kinetics. Moreover, CaMKII has been shown to regulate ethanol sensitivity of BK channels (Liu et al, 2006), raising the possibility that reduced levels of CaMKII could be acting more generally to modulate the ethanol sensitivity of channels in the BNST. Further, the regulation of CaMKII expression by alcohol exposure may be brain-region dependent, as a study in cultured cortical neurons found no effect of alcohol exposure on CaMKII levels (Xu et al, 2008).

Our finding that there is an upregulation of NR2B after chronic intermittent alcohol exposure is consistent with a number of earlier studies from a variety of systems, ranging from cell culture (Follesa and Ticku, 1996) to whole animals (Sircar and Sircar, 2006). In particular, our findings are similar to those reported in the closely related brain structure, CeA, of rats exposed to chronic continuous ethanol vapor (Roberto et al, 2004; Roberto et al, 2006). However, it is worth noting that the studies performed in the rat CeA also reported increases in NR1 and NR2A protein levels, whereas we failed to detect a change in levels of NR1 or NR2A in vBNST. This could be due in part to differences in exposure paradigms (continuous $v s$ intermittent), animal species used (rat vs mouse), or brainregion-specific differences in alcohol neuroadaptations. However, the concordance between our findings and the study from the CeA suggests that identification of the mechanisms that regulate alcohol-induced NR2B expression in the extended amygdala could lead to new treatments to regulate the severity of alcohol withdrawal.

On the basis of our results demonstrating an upregulation of NR2B, and our earlier work that showed that acute ethanol inhibition was NR2B-dependent in the vBNST we examined the ethanol sensitivity of NMDA EPSCs. We found that there was a decrease in the ability of ethanol to inhibit NMDA EPSCs in intermittent alcohol-exposed mice compared with control mice. One exciting interpretation of this result is that after this intermittent alcohol exposure paradigm, NMDA EPSCs in the BNST exhibit a tolerance to acute actions of ethanol. Further, this supports the assertion that acute ethanol sensitivity of NMDA-mediated synaptic currents are likely regulated by numerous factors in addition to subunit composition (Kash et al, 2008). This stands in contrast to the results obtained in rat CeA in which chronic ethanol treatment leads to an enhancement of the acute inhibitory actions of ethanol on NMDAmediated transmission (Roberto et al, 2004). The reason for this difference is unclear, perhaps relating to species and region-dependent alterations in ethanol-induced functional adaptations or to differences in the amount and duration of ethanol exposure.

We have shown earlier that acute ethanol exposure inhibits NMDAR function in the vBNST in an NR2Bdependent fashion (Kash et al, 2008). Taken together, this suggests that the upregulation of NR2B function in the vBNST is a homeostatic adaptation in response to the ability of acute alcohol exposure to inhibit NR2B-containing NMDARs in the vBNST. This would be in keeping with the model of alcohol-induced homeostatic plasticity proposed by Carpenter-Hyland and Chandler (CarpenterHyland et al, 2004). Briefly, Carpenter-Hyland and Chandler 
proposed that there is a homeostatic upregulation of synaptic NR2B-containing NMDAR in response to chronic alcohol inhibition, as we observed in the vBNST after intermittent alcohol exposure. Importantly, we found that in mice that were continuously exposed to alcohol vapor there was no alteration of temporal summation, suggesting a critical interaction between alcohol exposure and multiple withdrawals is required to elicit a change in function in the vBNST. It is not clear why the intermittent exposure is required for this change. One possibility is that there is a signaling event during withdrawal that alters function of the system, and with repeated withdrawals, this signaling event is sensitized leading to a more robust and long lasting change in function, resulting in the differences we observed. This is in keeping with several prominent theories regarding the etiology of alcoholism, namely the idea that multiple withdrawals can lead to allostatic shift in neuronal function through kindling or sensitization of key emotional circuits (Breese et al, 2005; Koob and Kreek, 2007).

One critical question that our findings raise is what is the impact of increased temporal summation of NMDARmediated responses on network function? Several studies have linked increased NR2B expression to a metaplastic shift in which it is easier to induce plasticity (Tang et al, 1999; Zhao et al, 2005; Philpot et al, 2007). Specifically, an increased temporal summation of NMDAR EPSCs in the visual cortex leads to a reduction in the stimulation frequency threshold required to induce NMDAR-dependent plasticity (Philpot et al, 2007). On the basis of similarity of our results to the findings in the visual cortex, one prediction would be that there would be a sensitization of NMDAR-dependent plasticity in the vBNST during acute withdrawal from intermittent alcohol exposure. The activity-dependent metaplasticity in the visual cortex after light deprivation was shown to be due to reduced cortical activation and a compensatory upregulation in the surface expression of NR2B NMDARs (Chen and Bear, 2007). Similarly, it is well accepted that the actions of ethanol are largely inhibitory in most regions of the CNS, including the vBNST. As the time-point in which we found a significant effect was $4-6 \mathrm{~h}$ postethanol vapor chamber exposure (alcohol exposure promoting CNS inhibition), the compensatory upregulation of the NR2B surface protein would effectively reduce the threshold for the induction of LTP. This shift in temporal summation and could lead to inappropriate recruitment and activation of regions downstream of the vBNST such as the LH and the PVN to what normally would be a sub-threshold stimulus, resulting in the altered behavior seen during alcohol withdrawal. It is critical to note that this alteration in function is transient, whereas many of the behavioral alterations after alcohol exposure are long lasting. This raises the possibility that early forms of plasticity such as observed in the vBNST can act to gate longer forms of plasticity in other regions of the brain.

\section{ACKNOWLEDGEMENTS}

This work was supported by NIH grants K99AA017668 (TLK), U01AA015635 (DGW), 5T32MH065215 (AJB), and RO1MH63232 (RJC), and a UNCF-Merck Postdoctoral fellowship (AJB). The authors would like to thank all the members of the Winder lab for helpful suggestions, specifically Zoe McElligott and Heather Gosnell for their comments on previous versions of the manuscript.

\section{DISCLOSURE/CONFLICT OF INTEREST}

The authors declare that, except for income received from primary employers, no financial support or compensation has been received from any individual or corporate entity over the past 3 years for research or professional service and there are no personal financial holdings that could be perceived as constituting a potential conflict of interest.

\section{REFERENCES}

Barria A, Malinow R (2005). NMDA receptor subunit composition controls synaptic plasticity by regulating binding to CaMKII. Neuron 48: 289-301.

Becker HC, Diaz-Granados JL, Weathersby RT (1997). Repeated ethanol withdrawal experience increases the severity and duration of subsequent withdrawal seizures in mice. Alcohol 14: 319-326.

Breese GR, Knapp DJ, Overstreet DH (2004). Stress sensitization of ethanol withdrawal-induced reduction in social interaction: inhibition by CRF-1 and benzodiazepine receptor antagonists and a 5-HT1A-receptor agonist. Neuropsychopharmacology 29: $470-482$.

Breese GR, Overstreet DH, Knapp DJ (2005). Conceptual framework for the etiology of alcoholism: a 'kindling'/stress hypothesis. Psychopharmacology (Berl) 178: 367-380.

Carpenter-Hyland EP, Chandler LJ (2006). Homeostatic plasticity during alcohol exposure promotes enlargement of dendritic spines. Eur J Neurosci 24: 3496-3506.

Carpenter-Hyland EP, Woodward JJ, Chandler LJ (2004). Chronic ethanol induces synaptic but not extrasynaptic targeting of NMDA receptors. J Neurosci 24: 7859-7868.

Chen WS, Bear MF (2007). Activity-dependent regulation of NR2B translation contributes to metaplasticity in mouse visual cortex. Neuropharmacology 52: 200-214.

Chu B, Anantharam V, Treistman SN (1995). Ethanol inhibition of recombinant heteromeric NMDA channels in the presence and absence of modulators. J Neurochem 65: 140-148.

Erreger K, Dravid SM, Banke TG, Wyllie DJ, Traynelis SF (2005). Subunit-specific gating controls rat NR1/NR2A and NR1/NR2B NMDA channel kinetics and synaptic signalling profiles. J Physiol 563: 345-358.

Follesa P, Ticku MK (1996). NMDA receptor upregulation: molecular studies in cultured mouse cortical neurons after chronic antagonist exposure. J Neurosci 16: 2172-2178.

Funk CK, O’Dell LE, Crawford EF, Koob GF (2006). Corticotropinreleasing factor within the central nucleus of the amygdala mediates enhanced ethanol self-administration in withdrawn, ethanol-dependent rats. J Neurosci 26: 11324-11332.

Gatch MB, Wallis CJ, Lal H (1999). Effects of NMDA antagonists on ethanol-withdrawal induced 'anxiety' in the elevated plus maze. Alcohol 19: 207-211.

Georges F, Aston-Jones G (2002). Activation of ventral tegmental area cells by the bed nucleus of the stria terminalis: a novel excitatory amino acid input to midbrain dopamine neurons. J Neurosci 22: 5173-5187.

Griffin 3rd WC, Lopez MF, Yanke AB, Middaugh LD, Becker HC (2009). Repeated cycles of chronic intermittent ethanol exposure in mice increases voluntary ethanol drinking and ethanol concentrations in the nucleus accumbens. Psychopharmacology (Berl) 201: 569-580, e-pub 2008 Sep 13. 
Healey JC, Winder DG, Kash TL (2008). Chronic ethanol exposure leads to divergent control of dopaminergic synapses in distinct target regions. Alcohol 42: 179-190.

Hendricson AW, Maldve RE, Salinas AG, Theile JW, Zhang TA, Diaz LM et al (2007). Aberrant synaptic activation of N-methylD-aspartate receptors underlies ethanol withdrawal hyperexcitability. J Pharmacol Exp Ther 321: 60-72.

Hoffman PL, Tabakoff B (1994). The role of the NMDA receptor in ethanol withdrawal. EXS 71: 61-70.

Kalluri HS, Mehta AK, Ticku MK (1998). Up-regulation of NMDA receptor subunits in rat brain following chronic ethanol treatment. Brain Res Mol Brain Res 58: 221-224.

Kash TL, Matthews RT, Winder DG (2008). Alcohol inhibits NR2B-containing NMDA receptors in the ventral bed nucleus of the stria terminalis. Neuropsychopharmacology 33: 1379-1390.

Kiefer F, Jahn H, Koester A, Montkowski A, Reinscheid RK, Wiedemann K (2003). Involvement of NMDA receptors in alcohol-mediated behavior: mice with reduced affinity of the NMDA R1 glycine binding site display an attenuated sensitivity to ethanol. Biol Psychiatry 53: 345-351.

Kliethermes CL, Cronise K, Crabbe JC (2004). Anxiety-like behavior in mice in two apparatuses during withdrawal from chronic ethanol vapor inhalation. Alcohol Clin Exp Res 28: 1012-1019.

Koob G, Kreek MJ (2007). Stress, dysregulation of drug reward pathways, and the transition to drug dependence. $A m J$ Psychiatry 164: 1149-1159.

Koob GF (2008). A role for brain stress systems in addiction. Neuron 59: 11-34.

Lister RG (1987). The use of a plus-maze to measure anxiey in the mouse. Psychopharmacology (Berl) 92: 180-185.

Liu J, Asuncion-Chin M, Liu P, Dopico AM (2006). CaM kinase II phosphorylation of slo Thr107 regulates activity and ethanol responses of BK channels. Nat Neurosci 9: 41-49.

Lovinger DM, White G, Weight FF (1989). Ethanol inhibits NMDAactivated ion current in hippocampal neurons. Science 243: 1721-1724.

McNeill RB, Colbran RJ (1995). Interaction of autophosphorylated $\mathrm{Ca} 2+/$ calmodulin-dependent protein kinase II with neuronal cytoskeletal proteins. Characterization of binding to a $190-\mathrm{kDa}$ postsynaptic density protein. J Biol Chem 270: 10043-10049.

Melendez RI, Hicks MP, Cagle SS, Kalivas PW (2005). Ethanol exposure decreases glutamate uptake in the nucleus accumbens. Alcohol Clin Exp Res 29: 326-333.

Mirshahi T, Woodward JJ (1995). Ethanol sensitivity of heteromeric NMDA receptors: effects of subunit assembly, glycine and NMDAR1 $\operatorname{Mg}(2+)$-insensitive mutants. Neuropharmacology 34: 347-355.

Overstreet DH, Knapp DJ, Breese GR (2002). Accentuated decrease in social interaction in rats subjected to repeated ethanol withdrawals. Alcohol Clin Exp Res 26: 1259-1268.

Philibin SD, Cameron AJ, Metten P, Crabbe JC (2008). Motor impairment: a new ethanol withdrawal phenotype in mice. Behav Pharmacol 19: 604-614.

Philpot BD, Cho KK, Bear MF (2007). Obligatory role of NR2A for metaplasticity in visual cortex. Neuron 53: 495-502.

Philpot BD, Sekhar AK, Shouval HZ, Bear MF (2001). Visual experience and deprivation bidirectionally modify the composi- tion and function of NMDA receptors in visual cortex. Neuron 29: $157-169$.

Roberto M, Bajo M, Crawford E, Madamba SG, Siggins GR (2006). Chronic ethanol exposure and protracted abstinence alter NMDA receptors in central amygdala. Neuropsychopharmacology 31: 988-996.

Roberto M, Schweitzer P, Madamba SG, Stouffer DG, Parsons LH, Siggins GR (2004). Acute and chronic ethanol alter glutamatergic transmission in rat central amygdala: an in vitro and in vivo analysis. J Neurosci 24: 1594-1603.

Sessoms-Sikes S, Honse Y, Lovinger DM, Colbran RJ (2005). CaMKllalpha enhances the desensitization of NR2B-containing NMDA receptors by an autophosphorylation-dependent mechanism. Mol Cell Neurosci 29: 139-147.

Sircar R, Sircar D (2006). Repeated ethanol treatment in adolescent rats alters cortical NMDA receptor. Alcohol 39: 51-58.

Spencer SJ, Buller KM, Day TA (2005). Medial prefrontal cortex control of the paraventricular hypothalamic nucleus response to psychological stress: possible role of the bed nucleus of the stria terminalis. J Comp Neurol 481: 363-376.

Strack S, McNeill RB, Colbran RJ (2000). Mechanism and regulation of calcium/calmodulin-dependent protein kinase II targeting to the NR2B subunit of the N-methyl-D-aspartate receptor. J Biol Chem 275: 23798-23806.

Suvarna N, Borgland SL, Wang J, Phamluong K, Auberson YP, Bonci A et al (2005). Ethanol alters trafficking and functional Nmethyl-D-aspartate receptor NR2 subunit ratio via H-Ras. J Biol Chem 280: 31450-31459.

Tang YP, Shimizu E, Dube GR, Rampon C, Kerchner GA, Zhuo M et al (1999). Genetic enhancement of learning and memory in mice. Nature 401: 63-69.

Valdez GR, Roberts AJ, Chan K, Davis H, Brennan M, Zorrilla EP et al (2002). Increased ethanol self-administration and anxietylike behavior during acute ethanol withdrawal and protracted abstinence: regulation by corticotropin-releasing factor. Alcohol Clin Exp Res 26: 1494-1501.

Walker DL, Davis M (2008). Role of the extended amygdala in short-duration versus sustained fear: a tribute to Dr. Lennart Heimer. Brain Struct Funct 213: 29-42. E-pub 2008 Jun 5.

Wang J, Carnicella S, Phamluong K, Jeanblanc J, Ronesi JA, Chaudhri $\mathrm{N}$ et al (2007). Ethanol induces long-term facilitation of NR2B-NMDA receptor activity in the dorsal striatum: implications for alcohol drinking behavior. J Neurosci 27: 3593-3602.

Xu M, Chandler LJ, Woodward JJ (2008). Ethanol inhibition of recombinant NMDA receptors is not altered by coexpression of CaMKII-alpha or CaMKII-beta. Alcohol 42: 425-432.

Yashiro K, Philpot BD (2008). Regulation of NMDA receptor subunit expression and its implications for LTD, LTP, and metaplasticity. Neuropharmacology 55: 1081-1094.

Yashiro K, Corlew R, Philpot BD (2005). Visual deprivation modifies both presynaptic glutamate release and the composition of perisynaptic/extrasynaptic NMDA receptors in adult visual cortex. J Neurosci 25: 11684-11692.

Zhao MG, Toyoda H, Lee YS, Wu LJ, Ko SW, Zhang XH et al (2005). Roles of NMDA NR2B subtype receptor in prefrontal long-term potentiation and contextual fear memory. Neuron 47: 859-872. 